\title{
Characterization of Phytophthora spp. Isolated from Ornamental Plants in Florida
}

\begin{abstract}
Jaimin S. Patel, Mid-Florida Research and Education Center and Department of Plant Pathology, University of Florida/Institute of Food and Agricultural Sciences, Apopka 32703; Anne Vitoreli and Aaron J. Palmateer, Tropical Research and Education Center, University of Florida/Institute of Food and Agricultural Sciences, Homestead 33031; Ashraf El-Sayed and David J. Norman, Mid-Florida Research and Education Center and Department of Plant Pathology, University of Florida/Institute of Food and Agricultural Sciences, Apopka; Erica M. Goss, Department of Plant Pathology, University of Florida, Institute of Food and Agricultural Sciences, Gainesville 32611-0680; and Mary S. Brennan and Gul Shad Ali, Mid-Florida Research and Education Center and Department of Plant Pathology, University of Florida/Institute of Food and Agricultural Sciences, Apopka
\end{abstract}

\begin{abstract}
Patel, J. S., Vitoreli, A., Palmateer, A. J., El-Sayed, A., Norman, D. J., Goss, E. M., Brennan, M. S., and Ali, G. S. 2016. Characterization of Phytophthora spp. isolated from ornamental plants in Florida. Plant Dis. 100:500-509.

This report investigates population structure and genetic variability of Phytophthora spp. isolated from botanically diverse plants in Florida. Internal transcribed spacer-based molecular phylogenetic analyses indicate that Phytophthora isolates recovered from ornamental plants in Florida represent a genetically diverse population and that a majority of the isolates belong to Phytophthora nicotianae (73.2\%), P. palmivora (18.7\%), $P$. tropicalis $(4.9 \%), P$. katsurae $(2.4 \%)$, and P. cinnamomi $(0.8 \%)$. Mating type analyses revealed that most isolates were heterothallic, consisting of both mating type A1 (25.2\%) and mating type A2 (39.0\%), and suggesting that they could outcross. Fungicide sensitivity assays

determined that several isolates were moderate to completely insensitive to mefenoxam. In addition, several isolates were also moderately insensitive to additional fungicides with different modes of action. However, correlation analyses did not reveal occurrence of fungicide cross-resistance. These studies suggest that a genetically diverse Phytophthora population infects ornamental crops and the occurrence of mefenoxam-insensitive Phytophthora populations raises concerns about disease management in ornamentals. Mitigating fungicide resistance will require prudent management strategies, including tank mixes and rotation of chemicals with different modes of actions.
\end{abstract}

Over 100 Phytophthora spp. have been described (Kroon et al. 2012), several of which infect economically important plants, including numerous vegetables, fruit, oil crops, ornamental plants, and landscape and forest trees (Erwin and Ribeiro 1996; Gevens et al. 2007; Grünwald et al. 2012; Martin et al. 2012). Worldwide, Phytophthora diseases are responsible for billions of dollars in crop losses (Kamoun et al. 2015; Wawra et al. 2012). Phytophthora spp. display substantial variation in host ranges, with some causing disease on a wider variety of plants whereas others infect only a single crop. They are regularly isolated from diverse ornamental plants in greenhouses and landscapes throughout the world (Bienapfl and Balci 2014; Leonberger et al. 2013; Parke et al. 2014; Prigigallo et al. 2015; Schlenzig et al. 2015). For example, Phytophthora nicotianae infects close to 255 genera in 90 families, including many herbaceous and woody plants, and is present in nurseries worldwide (Cline et al. 2008). P. nicotianae also causes root rot in citrus, resulting in substantial yield and quality losses where the disease occurs (Cacciola and Lio 2008). P. ramorum, which is an invasive and quarantined species, causes sudden oak death disease and infects over 60 ornamental trees and shrubs with devastating impacts on natural ecosystems (Grünwald et al. 2008, 2012). Another species, $P$. capsici, which was initially thought to infect pepper species only, was also found to infect cucurbits, tomato, eggplant, and bean (Martin et al. 2012; Wang et al. 2013). Common to the infection process of all Phytophthora spp. is the requirement of moist conditions, which

Corresponding author: G. S. Ali; E-mail: gsali@ufl.edu

J. S. Patel and A. Vitoreli contributed equally to this work.

*The $\boldsymbol{e}$-Xtra logo stands for "electronic extra" and indicates that one supplementary figure is published online.

Accepted for publication 2 September 2015.

http://dx.doi.org/10.1094/PDIS-05-15-0598-RE

(C) 2016 The American Phytopathological Society favors dispersal and germination of sporangia and zoospores. Long-distance dispersal of Phytophthora spp. is facilitated by run-off water from nurseries and by natural streams. The spread of Phytophthora spp. at a global level is believed to be through commercial trade of ornamentals (Bienapfl and Balci 2014; Goss et al. 2011b). For example, since the first detection of $P$. ramorum in the 1990s, it has spread to several U.S. states and Canada through shipments of infested ornamental plants (Goss et al. 2011b; Prospero et al. 2009; Rizzo et al. 2002).

Pesticides are the primary means of managing diseases caused by Phytophthora spp. (Foster and Hausbeck 2010), which add to the total cost of production. A number of fungicides with different modes of action such as phenyl amides, benzamides, phosphonate, dimethomorph, quinone outside inhibitors, and quinone inside inhibitors have been recommended to control diseases caused by Phytophthora spp. These fungicides are very effective because most of them inhibit single molecular sites in essential cellular processes such as mitochondrial function, ribosomal (r)RNA synthesis, cell wall biosynthesis, and cell division. Due to frequent use of single-site fungicides, Phytophthora spp. pose a high risk of developing resistance to fungicides and, therefore, potentially disrupting effective disease management programs (FRAC 2015; Hu et al. 2012). Fungicideinsensitive isolates of various Phytophthora spp. have been reported throughout the world (Dobrowolski et al. 2008; Gisi and Cohen 1996; Hu et al. 2005; Hu et al. 2012; Hu et al. 2008, 2010; Hwang and Benson 2005; Meng et al. 2011; Pérez-Sierra et al. 2011; Timmer et al. 1998). Therefore, regular monitoring of Phytophthora populations is essential for sustainable disease management (Brent 1992; Grünwald et al. 2006; Hu et al. 2012; Staub and Sozzi 1984).

Historically, Phytophthora spp. have been characterized through morphological features, which may not be practical for in-depth analyses of larger populations. More recently, several groups have used DNA-based molecular phylogenetic analyses for characterizing $P h y-$ tophthora communities in agricultural lands, nurseries, and natural ecosystems (Blair et al. 2008; Grünwald et al. 2011; Park et al. 2008). One of the first DNA regions to be used in phylogenetic analysis of Phytophthora spp. was the 5.8S rRNA gene and the flanking 
internal transcribed spacers (ITS) 1 and 2 (Lee and Taylor 1992). These studies have revealed important information about the genetic makeup, lineages, and spread of previously known and some newly discovered Phytophthora spp. throughout the world.

Several Phytophthora spp. are heterothallic, with two known mating types, type A1 and A2 (Gisi and Cohen 1996). Hybridization between opposite mating types increases genetic variability and promotes survival of the pathogen (Lamour and Hausbeck 2000). Sexual reproduction may also spread fungicide resistance through genetic recombination and, therefore, it is important to monitor mating types as well as fungicide sensitivity of Phytophthora spp.

Florida is home to thousands of commercial nurseries that produce a wide variety of ornamental plants ranging from annual herbaceous plants to tropical foliage and woody trees and shrubs (DPI 1998). Florida is the major port of entry for ornamental plants arriving in the United States (Merritt et al. 2012) and, subsequently, has a high number of pest and disease introductions. Phytophthora spp. are no exception and are frequently isolated from various ornamental plants in Florida. Based on morphological characteristics and previous pathological history, these isolates are tentatively assigned to specific species. However, molecular species identification is not routine. Accurate characterization of the genetic composition of the Phytophthora spp. infecting ornamentals is necessary for investigating the evolution, spread, and adaptation of Phytophthora spp. to new hosts and environments. Many commercial nurseries where these isolates were collected cocultivate many host plants of different Phytophthora spp., potentially increasing risk of development of hybrid species. Because the ornamental crop industry in Florida relies on frequent fungicide sprays for controlling diseases, the risks of selecting fungicide resistant Phytophthora isolates are high. The objectives of this study were to (i) conduct molecular phylogenetic analyses of a spatiotemporal population of Phytophthora recovered from ornamental crops in Florida, (ii) determine mating types of this population, and (iii) investigate the sensitivity of these Phytophthora spp. to commonly used fungicides with different modes of actions. Findings in this report will help in formulating effective strategies and policies for preventing the spread of Phytophthora spp. to new niches and mitigate risks of fungicide resistance development.

\section{Materials and Methods}

Phytophthora isolates. Between 1993 and 2014, in total, 156 putative Phytophthora isolates were recovered from the leading edges of lesions of different diseased plants with characteristics symptoms of Phytophthora diseases at the University of Florida Plant Disease Clinics at the Mid-Florida Research and Education Center, Apopka, FL and the Tropical Research and Education Center, Homestead, FL. These isolates were initially recovered on water agar and then stored in sterile water or $10 \% \mathrm{~V} 8$ juice agar slants in glass bottles at room temperature. For this study, we attempted to recover all these isolates on $10 \% \mathrm{~V} 8$, potato dextrose agar (PDA), and water agar. All cultures that grew were checked for any bacterial or fungal contamination, and contaminated cultures were excluded from further analysis, resulting in a population of 123 isolates (Table 1). A single hyphal tip from pure isolate cultures was transferred to and maintained on selective medium V8-PARP agar (20\% clarified V8 juice, $0.4 \%$ $\mathrm{CaCO}_{3}, 1.5 \%$ Bacto-agar, pimaricin at $25 \mu \mathrm{g} \mathrm{ml}{ }^{-1}$, ampicillin at $100 \mu \mathrm{g} \mathrm{ml}^{-1}$, rifampicin at $25 \mu \mathrm{g} \mathrm{ml}^{-1}$, and pentachloronitrobenzene at $25 \mu \mathrm{g} \mathrm{ml}^{-1}$ ). The first two digits in an isolate designation indicate year of collection. For this study, all isolates were grown on V8-PARP at $22^{\circ} \mathrm{C}$ for 6 to 8 days.

Polymerase chain reaction and sequencing of ITS. Approximately $100 \mathrm{mg}$ of mycelia of each isolate, grown on clarified $20 \%$ V8 PARP agar plates, was harvested by scraping them off with a sterile spatula into sterile 1.5-ml Eppendorf tubes. The mycelia were flash frozen with liquid nitrogen and ground using a pestle and mortar. DNA was isolated from the ground mycelia according to a previously described method (Aljanabi and Martinez 1997), and DNA was quantified using NanoDrop 2000C Spectrophotometer (Thermo Fisher Scientific). To accurately identify the isolates to species level, ITS regions were sequenced using the ITS5 forward primer and
ITS4 reverse primers to amplify the ITS1, 5.8s rRNA, and ITS2 regions (White et al. 1990) (Fig. 1). Polymerase chain reaction (PCR) consisted of $1 \times$ Ex Taq reaction buffer, $0.8 \mathrm{mM}$ all dNTPs, $0.3 \mu \mathrm{M}$ each primer, TaKaRa Ex Taq polymerase (TaKaRa) at $0.1 \mathrm{U} / \mu \mathrm{l}$, and genomic DNA at $0.8 \mathrm{ng} / \mu \mathrm{l}$ in a final volume of $25 \mu$ l. PCR conditions consisted of an initial denaturation step at $95^{\circ} \mathrm{C}$ for $5 \mathrm{~min}$ followed by 35 cycles of $95^{\circ} \mathrm{C}$ for $30 \mathrm{~s}, 55^{\circ} \mathrm{C}$ for $30 \mathrm{~s}$, and $72^{\circ} \mathrm{C}$ for $1 \mathrm{~min}$. PCR products were purified using the QIAquick PCR Purification Kit (Qiagen). Both strands of the amplified DNA products were directly sequenced using the ITS4 reverse and ITS5 forward primers. ITS sequences were searched against both the nonredundant nucleotide sequence database at the National Center for Biotechnology Information (NCBI; http://blast.ncbi.nlm.nih.gov/Blast.cgi) and the Phytophthora database (Kang et al. 2007).

Phylogenetic analysis. Genetic diversity of all isolates was determined using phylogenetic analyses. To infer genetic relationships of the Phytophthora isolates reported in this study, phylogenetic analyses were conducted using the ITS sequences. Representative ITS sequences of Phytophthora spp. were also included in constructing the phylogenetic tree for comparison. Sequences were aligned using ClustalW; alignment with the Muscle algorithm returned similar results. The ClustalW alignments were examined manually and ambiguous overhanging sequences from both ends were trimmed, resulting in an alignment block consisting of 772 sites, which spanned the ITS1, 5.8S rRNA, and ITS2 regions. For multiple sequence alignment, a FASTA file containing ITS sequences determined in this study and representative ITS sequences of Phytophthora spp. were imported into Mega 6.0 software (Larkin et al. 2007; Tamura et al. 2011) and aligned using the ClustalW algorithm (Larkin et al. 2007). Alignments were also performed using the Muscle algorithm (Edgar 2004) implemented in Mega 6.0. A phylogenetic tree was constructed using the maximumlikelihood (ML) method based on the Tamura-Nei model, with 1,000bootstrap replication (Tamura and Nei 1993; Tamura et al. 2011); the neighbor-joining method was also employed, which yielded results similar to the ML method. Evolutionary diversity estimates were calculated for the $P$. nicotianae group. Estimates of average evolutionary divergence over all isolate pairs were calculated as number of base substitutions per nucleotide site. Analyses were conducted using the maximum composite likelihood model (Tamura et al. 2004). All positions containing gaps and missing data were not included in analyses. There were 772 base positions in the final dataset.

Mating type analysis. Mating type of isolates was determined using known P. nicotianae mating type A1(number 13-724) and type A2 (number 13-723) tester strains in 24-well culture plates (Nunc; Thermo Fisher). Each well of the culture plate contained $0.5 \mathrm{ml}$ of V8 juice agar (20\% clarified V8 juice, $0.4 \% \mathrm{CaCO}_{3}$, and $1.5 \%$ Bacto-agar). A mycelial plug ( $2 \mathrm{~mm}$ in diameter) from a test isolate was placed at the margin of the well of a culture plate opposite a mycelial plug ( $2 \mathrm{~mm}$ in diameter) of either mating type A1 or A2. Each isolate was also tested for homothallism using two agar plugs from the same isolate. Plates were wrapped with Parafilm and incubated in a growth chamber at 22 to $25^{\circ} \mathrm{C}$ for up to 6 weeks under dark. Plates were checked for the presence of oospores every week for at least 6 weeks.

Fungicide sensitivity tests. Most isolates were tested for sensitivity to mefenoxam (Fungicide Resistance Action Committee [FRAC] group 4, Subdue Maxx; Syngenta Crop Protection), fluopicolide (FRAC group 43, Adorn; Valent USA Corporation), aluminum Tris (FRAC group 33, Aliette WDG; Bayer Crop Science), azoxystrobin (FRAC group 11, Heritage; Syngenta Crop Protection), cyazofamid (FRAC group 21, Segway; FMC Corporation), and dimethomorph (FRAC group 40, Stature SC; BASF). Fungicides formulated as commercial products were dissolved in autoclaved clarified V8 medium at the following active ingredient rates: mefenoxam, $0.1 \mathrm{mg} \mathrm{ml}^{-1}$; fluopicolide, $0.1 \mathrm{mg} \mathrm{ml}^{-1}$; aluminum Tris, $1 \mathrm{mg} \mathrm{ml}^{-1}$; azoxystrobin, $1 \mathrm{mg} \mathrm{ml}^{-1}$; cyzofamid, $1 \mathrm{mg} \mathrm{ml}^{-1}$; and dimethomorph, $1 \mathrm{mg} \mathrm{ml}^{-1}$. Fungicide concentrations chosen in this study were based on previous studies (Jackson et al. 2010; Kousik and Keinath 2008; Kuhajek et al. 2003; Stein and Kirk 2004; Ziogas et al. 2006). A single 5-mm 
Table 1. Isolates of Phytophthora spp. collected from various ornamental hosts in Florida

\begin{tabular}{|c|c|c|c|c|c|c|}
\hline Species $^{\mathbf{a}}$ & Isolates & ITS identity $(\%)^{\mathbf{b}}$ & Host & \multicolumn{2}{|c|}{ Mating types ${ }^{\mathrm{c}}$} & Mefenoxam sensitivity ${ }^{d}$ \\
\hline cinnamomi & $07-385$ & 99.9 & Rhododendron sp. & $\ldots$ & A2 & $\mathrm{S}$ \\
\hline katsurae & 14-374B & 99.0 & Podocarpus sp. & $\mathrm{A} 1 \mathrm{~A} 2$ & $\mathrm{~A} 1 \mathrm{~A} 2$ & $\mathrm{~S}$ \\
\hline katsurae & $14-374 \mathrm{C}$ & 99.0 & Podocarpus sp. & $\mathrm{A} 1 \mathrm{~A} 2$ & $\mathrm{~A} 1 \mathrm{~A} 2$ & $\mathrm{~S}$ \\
\hline katsurae & $14-761$ & 99.0 & Podocarpus sp. & $\mathrm{A} 1 \mathrm{~A} 2$ & $\mathrm{~A} 1 \mathrm{~A} 2$ & $\mathrm{~S}$ \\
\hline palmivora & 01-118 & 100.0 & Hedera sp. & $\ldots$ & $\mathrm{A} 2$ & $\mathrm{~S}$ \\
\hline palmivora & $03-550$ & 99.4 & Chamaedorea elegans & A1 & $\ldots$ & $\mathrm{S}$ \\
\hline palmivora & $11-65$ & 99.9 & Spathiphyllum sp. & $\ldots$ & A2 & $\mathrm{S}$ \\
\hline palmivora & $10-1733$ & 99.0 & Liriope muscari & - & - & $\mathrm{S}$ \\
\hline palmivora & $13-1483 \mathrm{~A}$ & 100.0 & Chrysothemis sp. & - & - & $\mathrm{S}$ \\
\hline palmivora & 13-1483B & 100.0 & Chrysothemis sp. & - & - & $\mathrm{S}$ \\
\hline palmivora & $13-1726$ & 100.0 & Mandevilla $\mathrm{sp}$. & - & - & $\mathrm{S}$ \\
\hline palmivora & $13-1734$ & 98.0 & Pheonix dactylifera & - & - & $\ldots$ \\
\hline palmivora & $13-1735$ & 97.0 & Catharanthus sp. & - & - & $\ldots$ \\
\hline palmivora & $14-130$ & 100.0 & Cordyline sp. & $\ldots$ & $\mathrm{A} 2$ & $\mathrm{~S}$ \\
\hline palmivora & $14-32$ & 100.0 & Latania sp. & - & - & $\mathrm{S}$ \\
\hline palmivora & $14-33$ & 100.0 & Latania sp. & - & - & $\mathrm{S}$ \\
\hline palmivora & $14-34$ & 99.0 & Latania sp. & - & - & $\mathrm{S}$ \\
\hline palmivora & $14-35$ & 100.0 & Latania sp. & - & - & $\mathrm{S}$ \\
\hline palmivora & $14-37$ & 99.0 & Latania sp. & - & - & $\mathrm{S}$ \\
\hline palmivora & $14-684 \mathrm{~A}$ & 100.0 & Podocarpus sp. & - & - & $\mathrm{S}$ \\
\hline palmivora & 14-684B & 99.0 & Sabal palmetto & - & - & $\mathrm{S}$ \\
\hline palmivora & $14-704$ & 97.0 & Pothos sp. & - & - & $\mathrm{S}$ \\
\hline palmivora & $14-705$ & 97.0 & Pothos sp. & - & - & $\mathrm{S}$ \\
\hline palmivora & 14-708 & 99.0 & Palm & $\cdots$ & $\mathrm{A} 2$ & $\mathrm{~S}$ \\
\hline palmivora & 14-709 & 99.0 & Palm & $\ldots$ & A2 & $\mathrm{S}$ \\
\hline palmivora & $14-710$ & 98.0 & Pachira sp. & - & - & $\mathrm{S}$ \\
\hline palmivora & $14-711$ & 98.0 & Pachira sp. & - & - & $\mathrm{S}$ \\
\hline nicotianae & $93-653$ & 99.9 & Vinca sp. & $\ldots$ & A2 & $\mathrm{S}$ \\
\hline nicotianae & 99-191 & 99.9 & Spathiphyllum sp. & $\ldots$ & $\mathrm{A} 2$ & $\mathrm{~S}$ \\
\hline nicotianae & $00-334$ & 100.0 & Vinca $\mathrm{sp}$ & A1 & $\ldots$ & $\mathrm{S}$ \\
\hline nicotianae & $01-138$ & 98.4 & Spathiphyllum sp. & $\ldots$ & A2 & $\mathrm{S}$ \\
\hline nicotianae & $01-280$ & 99.7 & Anthurium sp. & $\cdots$ & $\mathrm{A} 2$ & $\mathrm{~S}$ \\
\hline nicotianae & 01-302 & 99.3 & Viola sp. & - & - & $\mathrm{S}$ \\
\hline nicotianae & $02-29$ & 99.1 & Calceolaria sp. & $\ldots$ & A2 & $\mathrm{S}$ \\
\hline nicotianae & $03-131$ & 99.9 & Spathiphyllum sp. & $\ldots$ & $\mathrm{A} 2$ & $\mathrm{~S}$ \\
\hline nicotianae & 03-132 & 100.0 & Spathiphyllum sp. & - & - & $\mathrm{S}$ \\
\hline nicotianae & $03-361$ & 100.0 & Dieffenbachia sp. & A1 & - & $\mathrm{S}$ \\
\hline nicotianae & 03-407 & 99.3 & Pothos sp. & A1 & - & $\mathrm{S}$ \\
\hline nicotianae & 04-130 & 99.9 & Spathiphyllum sp. & A1 & $\ldots$ & $\mathrm{S}$ \\
\hline nicotianae & $04-325$ & 100.0 & Spathiphyllum sp. & $\ldots$ & A2 & $\mathrm{S}$ \\
\hline nicotianae & $04-410$ & 99.8 & Spathiphyllum sp. & $\ldots$ & $\mathrm{A} 2$ & $\mathrm{~S}$ \\
\hline nicotianae & $05-215$ & 99.6 & Alocasia sp. & $\ldots$ & A2 & $\mathrm{S}$ \\
\hline nicotianae & $05-217$ & 100.0 & Coleus sp. & - & - & $\mathrm{S}$ \\
\hline nicotianae & $05-222$ & 99.6 & Gerbera sp. & $\ldots$ & $\mathrm{A} 2$ & $\mathrm{~S}$ \\
\hline nicotianae & $05-287$ & 97.2 & Pothos sp. & $\ldots$ & A2 & $\mathrm{S}$ \\
\hline nicotianae & $05-297$ & 98.5 & Poinsettia & $\ldots$ & $\mathrm{A} 2$ & $\mathrm{R}$ \\
\hline nicotianae & 06-141 & 99.9 & Spathiphyllum sp. & $\ldots$ & $\mathrm{A} 2$ & $\mathrm{~S}$ \\
\hline nicotianae & $06-174$ & 100.0 & Cattleya sp. & $\ldots$ & $\mathrm{A} 2$ & $\mathrm{~S}$ \\
\hline nicotianae & $07-57$ & 99.8 & Alocasia sp. & A1 & $\ldots$ & $\mathrm{S}$ \\
\hline nicotianae & $08-168$ & 99.7 & Spathiphyllum sp. & - & - & $\mathrm{S}$ \\
\hline nicotianae & $10-43$ & 99.9 & Spathiphyllum sp. & A1 & $\ldots$ & $\mathrm{S}$ \\
\hline nicotianae & $11-1$ & 99.9 & Spathiphyllum sp. & $\ldots$ & $\mathrm{A} 2$ & $\mathrm{~S}$ \\
\hline nicotianae & $11-3$ & 100.0 & Spathiphyllum sp. & - & - & $\mathrm{S}$ \\
\hline nicotianae & $11-21$ & 99.0 & Rhoeo discolor & $\cdots$ & $\mathrm{A} 2$ & $\mathrm{~S}$ \\
\hline nicotianae & $11-38$ & 100.0 & Impatiens sp. & $\ldots$ & $\mathrm{A} 2$ & $\mathrm{~S}$ \\
\hline nicotianae & $12-1$ & 98.6 & Spathiphyllum sp. & $\cdots$ & $\mathrm{A} 2$ & $\mathrm{~S}$ \\
\hline nicotianae & $12-5$ & 98.2 & Hibiscus sp. & $\ldots$ & $\mathrm{A} 2$ & $\mathrm{~S}$ \\
\hline nicotianae & $10-524 \mathrm{~A}$ & 99.0 & Iris sp. & $\ldots$ & $\mathrm{A} 2$ & $\mathrm{~S}$ \\
\hline nicotianae & $10-524 B$ & 99.0 & Iris sp. & $\ldots$ & $\mathrm{A} 2$ & $\mathrm{~S}$ \\
\hline nicotianae & 10-534B & 100.0 & Spathiphyllum sp. & - & - & $\mathrm{S}$ \\
\hline & & & & & & (continued on next page) \\
\hline
\end{tabular}

a Phytophthora spp. were identified based on BLAST match in the National Center for Biotechnology Information database and Phytophthora database.

b Percent identity to ITS sequences in the Phytophthora database.

c A1 and A2 mating types were determined using known tester mating type A1 (P. nicotianae \#13-724) and A2 (P. nicotianae \#13-723); each isolate was also tested with itself for homothallism; - indicates sterile, defined as those that did not display any evidence of oospores, antheridia, or oogonia; and A1A2 represents homothallic isolates.

d $\mathrm{S}$ and $\mathrm{R}$ indicate sensitive and resistant isolates, respectively, which were defined as those that displayed $\geq 80 \%$ (S) or $\leq 50 \%$ (R) growth inhibition on $\mathrm{V} 8$ agar plates containing mefenoxam at $100 \mu \mathrm{g} \mathrm{m} \mathrm{m}^{-1}$. 
Table 1. (continued from preceding page)

\begin{tabular}{|c|c|c|c|c|c|c|}
\hline Species $^{\mathbf{a}}$ & Isolates & ITS identity $(\%)^{b}$ & Host & & $\operatorname{pes}^{c}$ & Mefenoxam sensitivity ${ }^{d}$ \\
\hline nicotianae & $10-616 \mathrm{~A}$ & 100.0 & Spathiphyllum sp. & - & - & $\mathrm{S}$ \\
\hline nicotianae & $10-616 \mathrm{~B}$ & 99.0 & Spathiphyllum sp. & - & - & $\mathrm{S}$ \\
\hline nicotianae & $10-616 \mathrm{C}$ & 100.0 & Unknown & - & - & $\mathrm{S}$ \\
\hline nicotianae & $13-1133 \mathrm{~A}$ & 100.0 & Mandevilla sp. & - & - & $\mathrm{S}$ \\
\hline nicotianae & 13-1134B & 100.0 & Rhododendron sp. & A1 & $\ldots$ & $\mathrm{S}$ \\
\hline nicotianae & $13-1667$ & 100.0 & Mandevilla $\mathrm{sp}$. & - & - & $\mathrm{S}$ \\
\hline nicotianae & $13-1727$ & 100.0 & Mandevilla sp. & - & - & $\mathrm{S}$ \\
\hline nicotianae & $13-1728$ & 99.0 & Mandevilla sp. & - & - & $\mathrm{S}$ \\
\hline nicotianae & $13-1729$ & 100.0 & Allamanda sp. & - & - & $\mathrm{S}$ \\
\hline nicotianae & $13-1730$ & 100.0 & Spathiphyllum sp. & - & - & $\mathrm{S}$ \\
\hline nicotianae & $13-1731$ & 100.0 & Spathiphyllum sp. & - & - & $\mathrm{S}$ \\
\hline nicotianae & $13-1732$ & 100.0 & Spathiphyllum sp. & A1 & $\ldots$ & $\mathrm{S}$ \\
\hline nicotianae & $13-1815$ & 100.0 & Kalanchoe sp. & $\ldots$ & $\mathrm{A} 2$ & $\mathrm{~S}$ \\
\hline nicotianae & $13-1816$ & 100.0 & Not determined & $\ldots$ & $\mathrm{A} 2$ & $\mathrm{~S}$ \\
\hline nicotianae & $13-1817$ & 100.0 & Not determined & A1 & $\ldots$ & $\mathrm{S}$ \\
\hline nicotianae & $13-1818$ & 99.0 & Swietenia sp. & - & - & $\mathrm{S}$ \\
\hline nicotianae & $13-1819$ & 100.0 & Strelitzia nicolai & $\ldots$ & $\mathrm{A} 2$ & $\mathrm{~S}$ \\
\hline nicotianae & $13-1820$ & 100.0 & Bursera sp. & A1 & $\ldots$ & $\mathrm{S}$ \\
\hline nicotianae & $13-1821$ & 99.0 & Syagrus romanzoffiana & $\ldots$ & $\mathrm{A} 2$ & $\mathrm{~S}$ \\
\hline nicotianae & $13-1822$ & 100.0 & Casia $\mathrm{sp}$. & A1 & $\ldots$ & $\mathrm{S}$ \\
\hline nicotianae & $13-1823$ & 100.0 & Casia sp. & A1 & $\ldots$ & $\mathrm{S}$ \\
\hline nicotianae & $13-1824$ & 99.0 & Impatiens sp. walleriana & $\ldots$ & $\mathrm{A} 2$ & $\mathrm{~S}$ \\
\hline nicotianae & $13-1825$ & 100.0 & Impatiens sp. walleriana & A1 & $\ldots$ & $\mathrm{S}$ \\
\hline nicotianae & $13-1826$ & 99.0 & Croton sp. & $\ldots$ & A2 & $\mathrm{S}$ \\
\hline nicotianae & $13-1827$ & 100.0 & Oncidium sp. & A1 & $\ldots$ & $\mathrm{S}$ \\
\hline nicotianae & $13-1828$ & 100.0 & Catharanthus sp. & A1 & $\ldots$ & $\mathrm{S}$ \\
\hline nicotianae & $13-1829$ & 100.0 & Catharanthus sp. & A1 & $\ldots$ & $\mathrm{S}$ \\
\hline nicotianae & $13-1830$ & 100.0 & Catharanthus sp. & A1 & $\ldots$ & $\mathrm{S}$ \\
\hline nicotianae & $13-1831$ & 100.0 & Tilandsia sp. & A1 & $\ldots$ & $\mathrm{S}$ \\
\hline nicotianae & $13-1832$ & 100.0 & Dracaena sp. & A1 & $\ldots$ & $\mathrm{S}$ \\
\hline nicotianae & $13-1833$ & 100.0 & Dracaena sp. & A1 & $\ldots$ & $\mathrm{S}$ \\
\hline nicotianae & $13-1834$ & 100.0 & Schefflera sp. & A1 & $\ldots$ & $\mathrm{S}$ \\
\hline nicotianae & $13-1835$ & 99.0 & Schefflera sp. & A1 & $\ldots$ & $\mathrm{S}$ \\
\hline nicotianae & $13-1836$ & 100.0 & Dracaena sp. & A1 & $\ldots$ & $\mathrm{S}$ \\
\hline nicotianae & $13-1837$ & 100.0 & Anthurium sp. & - & - & $\mathrm{S}$ \\
\hline nicotianae & $13-1838$ & 99.0 & Sansevieria sp. & A1 & $\ldots$ & $\mathrm{S}$ \\
\hline nicotianae & $13-1839$ & 100.0 & Epipremnum sp. & A1 & $\ldots$ & $\mathrm{S}$ \\
\hline nicotianae & $13-1840$ & 99.0 & Mandevilla sp. & $\ldots$ & $\mathrm{A} 2$ & $\mathrm{~S}$ \\
\hline nicotianae & $13-1841$ & 100.0 & Philodendrun & $\ldots$ & $\mathrm{A} 2$ & $\mathrm{~S}$ \\
\hline nicotianae & $13-1842$ & 99.0 & Epipremnum sp. & $\ldots$ & $\mathrm{A} 2$ & $\mathrm{~S}$ \\
\hline nicotianae & $13-1843$ & 99.0 & Adenium sp. & $\ldots$ & A2 & $\mathrm{S}$ \\
\hline nicotianae & $13-1844$ & 100.0 & Mandevilla sp. & $\ldots$ & $\mathrm{A} 2$ & $\mathrm{~S}$ \\
\hline nicotianae & $13-1845$ & 100.0 & Geranium sp. & $\ldots$ & $\mathrm{A} 2$ & $\mathrm{~S}$ \\
\hline nicotianae & $14-1030$ & 99.0 & Anthurium sp. & - & - & $\ldots$ \\
\hline nicotianae & $14-1585 B$ & 99.0 & Gardenia sp. & - & - & $\mathrm{S}$ \\
\hline nicotianae & $14-1585 \mathrm{C}$ & 99.0 & Gardenia sp. & - & - & $\mathrm{S}$ \\
\hline nicotianae & $14-214 \mathrm{~A}$ & 100.0 & Phoenix sp. & $\ldots$ & A2 & $\mathrm{S}$ \\
\hline nicotianae & 14-214B & 100.0 & Gardenia sp. & $\ldots$ & $\mathrm{A} 2$ & $\mathrm{~S}$ \\
\hline nicotianae & $14-215$ & 100.0 & Gardenia sp. & $\ldots$ & A2 & $\mathrm{S}$ \\
\hline nicotianae & $14-271 \mathrm{~A}$ & 98.0 & Podocarpus sp. & $\ldots$ & $\mathrm{A} 2$ & $\mathrm{~S}$ \\
\hline nicotianae & $14-707$ & 99.0 & Pothos sp. & $\ldots$ & $\mathrm{A} 2$ & $\mathrm{~S}$ \\
\hline nicotianae & $14-712$ & 99.0 & Spathiphyllum sp. & A1 & $\ldots$ & $\mathrm{S}$ \\
\hline nicotianae & $14-754 \mathrm{~A}$ & 99.0 & Spathiphyllum sp. & A1 & $\ldots$ & $\mathrm{S}$ \\
\hline nicotianae & 14-754B & 99.0 & Spathiphyllum sp. & - & - & $\mathrm{S}$ \\
\hline nicotianae & $14-754 \mathrm{C}$ & 99.0 & Spathiphyllum sp. & A1 & $\ldots$ & $\mathrm{S}$ \\
\hline nicotianae & $14-773 \mathrm{~A}$ & 99.0 & Strelitzia reginae & $\ldots$ & $\mathrm{A} 2$ & $\mathrm{~S}$ \\
\hline nicotianae & $14-820$ & 99.0 & Petunia sp. & $\ldots$ & $\mathrm{A} 2$ & $\mathrm{~S}$ \\
\hline tropicalis & $96-83$ & 100.0 & Hedera sp. & A1 & $\ldots$ & $\mathrm{S}$ \\
\hline tropicalis & $03-387$ & 100.0 & Hedera sp. & $\ldots$ & $\mathrm{A} 2$ & $\mathrm{~S}$ \\
\hline tropicalis & $03-452$ & 100.0 & Hedera sp. & A1 & - & $\mathrm{S}$ \\
\hline tropicalis & $08-228$ & 99.8 & Hedera sp. & $\ldots$ & $\mathrm{A} 2$ & $\mathrm{~S}$ \\
\hline tropicalis & $14-138$ & 99.0 & Pepper & - & - & $\mathrm{R}$ \\
\hline tropicalis & $14-822 \mathrm{~A}$ & 99.0 & Rosmarinus officinalis & - & - & $\mathrm{S}$ \\
\hline
\end{tabular}


mycelial plug from the leading edge of a 1-week-old Phytophthora culture, grown in the dark at $22^{\circ} \mathrm{C}$, was placed on fungicideamended and nonamended clarified V8 agar plates. Plates were incubated in the dark at 22 to $25^{\circ} \mathrm{C}$ for 7 days. Colony diameters were measured and percent growth inhibition was calculated using the following formula:

$$
\text { Percent growth inhibition }=\frac{C-F}{C} \times 100
$$

where $C$ and $F$ are average colony diameters on nonamended control and fungicide-amended V8 agar plates, respectively. To determine the effect of mefenoxam dosage on resistance, isolate 05-297 (resistant, with less than 10\% growth inhibition) and isolate 05-217 (sensitive, with $>90 \%$ growth inhibition) were assayed over a range of mefenoxam concentrations. All experiments were arranged in a completely randomized design with four replications for each fungicide. The experiment was repeated once. The emergence of fungicide-resistant populations has been reported in several Phytophthora spp. (Dobrowolski et al. 2008; Gisi and Cohen 1996; Hu et al. 2005; Hu et al. 2012; $\mathrm{Hu}$ et al. 2008, 2010; Hwang and Benson 2005; Meng et al. 2011; Pérez-Sierra et al. 2011; Timmer et al. 1998) and poses serious threat to effective management of Phytophthora diseases. Therefore, Phytophthora isolates collected in this study were screened against commonly used fungicides. Based on the percent growth inhibition, isolates were categorized into sensitive ( $\geq 80 \%$ growth inhibition), moderately sensitive (51 to $79 \%$ growth inhibition), and resistant ( $\leq 50 \%$ growth inhibition). Cross-resistance of isolates to different fungicides was analyzed using the Spearman's rank correlation coefficients $(\rho)$ on ranked percent growth inhibition data. Spearman's $\rho$ values and their associated $P$ values were calculated using the open source statistical software R (http:// www.r-project.org/).

\section{Results}

Molecular identification of Phytophthora isolates. Consensus ITS contigs for each isolate were constructed from the alignment of forward and reverse sequences obtained with ITS5 and IT4 primers. The resulting contigs were searched using BLASTn at NCBI (http://blast.ncbi.nlm.nih.gov/Blast.cgi) and at the manually curated Phytophthora database (Park et al. 2008). BLAST search results from NCBI and the Phytophthora database were identical for each isolate (Table 1). Among the 123 isolates, 90 isolates were identified as $P$. nicotianae, 23 as $P$. palmivora, 6 as $P$. tropicalis, 3 as $P$. katsurae, and 1 as P. cinnamomi (Table 1). Because double peaks were not observed in the DNA sequence chromatograms of any of the isolates, this suggested that these isolates are homozygous for ITS.

Diversity and genetic composition of Phytophthora isolates. ClustalW alignment showed that approximately 33\% (252/772) of the sites were polymorphic. An ML tree was constructed from these alignments, which separated all isolates into five major groups, consistent with BLAST results, such that each group closely clustered with a representative published ITS sequence of $P$. parasitica

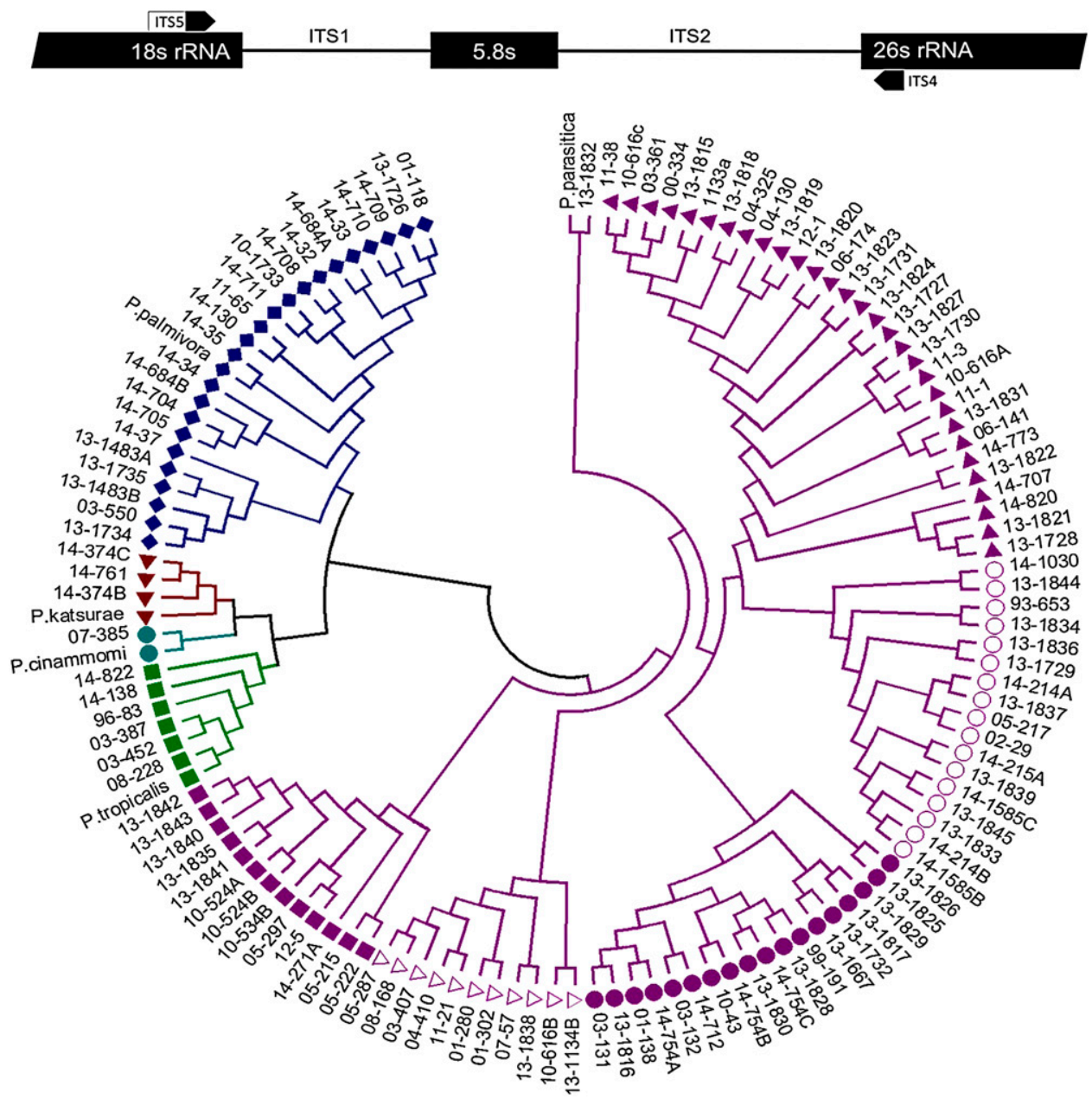

Fig. 1. Molecular phylogenetic analysis of Phytophthora isolates. Upper panel shows schematics of the internal transcribed spacer (ITS) region of Phytophthora spp. Locations of ITS4 and ITS5 primers are indicated. Lower panel shows the phylogenetic tree, which was constructed from the ITS sequences using the maximum-likelihood method. Analysis consisted of 128 sequences including five reported representative ITS sequence of P. nicotianae (GU983635), P. palmivora (KF263691), P. tropicalis (KC479199), P. katsurae (JQ901391), and P. cinnamomi (JX996044). 
(GU983635), P. palmivora (KF263691), P. tropicalis (KC479199), P. katsurae (JQ901391), and P. cinnamomi (JX996044) (Fig. 1). Using the maximum composite likelihood model (Tamura et al. 2004), evolutionary diversity estimate, calculated as mean base substitution per site over all sequence pairs, was $0.0286(2.86 \%)$.

The $P$. nicotianae group, which consisted of 90 isolates, displayed substantial variation and was further analyzed in detail for genetic diversity. These analyses classified the $P$. nicotianae isolates roughly into five subgroups (Fig. 1). Overall, mean divergence between $P$. nicotianae isolates was $0.64 \%$ (range, 0 to $7.5 \%$ ). In pairwise comparisons, a majority of the $P$. nicotianae isolates displayed mean divergence below $2 \%$. These isolates infected plants from diverse families. Groups $P p I V$ and $P p V$ displayed high within-group and between-group divergences (Tables 2 and 3). Similarly, $P$. palmivora isolates also displayed considerable variation in ITS sequence, with a $0.44 \%$ (range, 0 to $2.3 \%$ ) overall mean divergence.

Phytophthora isolates are a mixture of $\mathrm{A} 1$ and $\mathrm{A} 2$ mating types. Mating types of all isolates were identified using known A1 and A2 mating type tester isolates. A summary of this analysis is presented in Table 4. Overall, $25.2 \%$ of all Phytophthora isolates belonged to mating type A1 and $39.0 \%$ belonged to mating type A2. The three isolates identified as P. katsurae were all homothallic and produced oospores. The remaining $33.3 \%$ did not develop any oospores, antheridia, or oogonia during up to 6 weeks of culture, suggesting that they are sterile.

Several Phytophthora isolates displayed mefenoxam-insensitivity. A majority of isolates were either sensitive (53\%) or moderately sensitive $(37 \%)$ to mefenoxam, suggesting that they can be controlled using mefenoxam. There was substantial variation in mefenoxam sensitivities of these isolates (Supplementary Fig. S1). One P. tropicalis and three $P$. nicotianae isolates were found to be resistant to mefenoxam. Among these, isolate 05-297 (P. nicotianae) was highly resistant to mefenoxam, with only $9.4 \%$ growth inhibition (Fig. 2). The other three isolates, 05-215 (P. nicotianae), 11-38 (P. nicotianae), and 14-138 ( $P$. tropicalis) had 43.7, 47.7, and $23.9 \%$ growth reduction, respectively. Isolate 05-297 displayed a high level of resistance over all tested concentrations. Interestingly, at sublethal concentrations ( 0.1 to $\left.2 \mu \mathrm{g} \mathrm{ml}^{-1}\right)$, the growth of this isolate was enhanced by approximately $20 \%$, which was observed consistently in several independent experiments (Fig. 2A). Similar results were observed with the other three resistant isolates (05-215, 11-38, and 14-138; data not shown). Because mefenoxam is used extensively in nurseries, the resistant strains will likely be selected, thus increasing the probability of mefenoxam-resistance build-up in future Phytophthora populations.

Phytophthora isolates did not display any resistance to different fungicides. A majority of the tested isolates were sensitive to fluopicolide (Adorn), aluminum Tris (Aliette), azoxystrobin (Heritage), cyzofamid (Segway), and dimethomorph (Stature). Frequency distribution of sensitive, moderately resistant, and resistant isolates is shown in Figure 3. More than $80 \%$ of isolates were sensitive to mefenoxam, Adorn, and Stature (Fig. 3). Although a majority of isolates were inhibited by each of these fungicides, several isolates displayed variable resistance levels to at least one of these fungicides. Less than
$50 \%$ growth inhibition was displayed by 1 (Adorn), 9 (Aliette), 10 (Heritage), and 11 (Segway) isolates (Fig. 3). The remaining isolates either displayed moderate resistance or were sensitive to these fungicides. Ornamental facilities, where the isolates reported in this study originated, often apply fungicides with different modes of action on the same crop multiple times. This led us to speculate that some isolates might display resistance to multiple fungicides. With the exception of a very low-level and statistically insignificant coresistance between mefenoxam and Adorn $(\rho=0.31, P=0.09)$ and between mefenoxam and Aliette $(\rho=0.30, P=0.09)$, none of the other fungicides showed resistance to multiple isolates. All isolates which were resistant to one fungicide were inhibited by at least one other fungicide with a different mode of action. For example, isolates $05-215,05-217$, and 05-222 showed moderate resistance to Adorn but were sensitive to Aliette, Heritage, and Stature.

No correlation was found between genetic diversity of isolates and their sensitivity to fungicides (Table 2; Figs. 2 and 3; Spearmans's rank correlation $P>0.1$ ). For example, isolates 05-297 (resistant to mefenoxam) and 12-5 (sensitive to mefenoxam), belong to the same group $P p V$ in the phylogenetic tree (Fig. 1). Isolates 05-297, 05-215, and 11-38, which were mefenoxam-resistant, displayed singlenucleotide polymorphism (SNP) at different sites and belonged to different phylogenetic groups (Fig. 1), suggesting that mefenoxam insensitivity is not necessarily associated with overall genetic variation.

\section{Discussion}

Florida is renowned for its ornamental plant industry and it ranks second only to California in total trade. Altogether, thousands of ornamental plant species are grown throughout Florida in greenhouses and nurseries. Ornamental plant producers in Florida often face heavy pressure from pathogens and insects which, due to a hot and humid climate, can be a year-round challenge. In addition, every year, millions of plants are traded through Florida, which can serve as an unintentional conduit for the spread of pathogens to new areas, where they can infect resident plants. Conglomeration of so many diverse plants and their associated pathogens in confined greenhouses can also serve as a potential breeding ground for the evolution

Table 3. Estimates of average evolutionary divergence over sequence pairs between groups ${ }^{\mathrm{a}}$

\begin{tabular}{lc}
\hline Group & Within-group distance \\
\hline$P p I$ & 0.00017 \\
$P p$ II & 0.00010 \\
$P p$ III & 0.00009 \\
$P p$ IV & 0.00216 \\
$P p$ V & 0.01073 \\
Phytophthora palmivora & 0.02053 \\
P. tropicalis & 0.00433 \\
P. katsurae & 0.00197 \\
\hline
\end{tabular}

a PpI, PpII, PpIII, PpIV, and $P p V$ are subgroups of $P$. nicotianae. The number of base substitutions per site from averaging over all sequence pairs within each group are shown. Analysis involved 128 nucleotide sequences.

Table 2. Estimates of average evolutionary divergence over sequence pairs between groups ${ }^{\mathrm{a}}$

\begin{tabular}{|c|c|c|c|c|c|c|c|c|}
\hline & $P p I I$ & $P p ~ I I I$ & $P p I V$ & $P p V$ & P. palmivora & P. tropicalis & P. katsurae & P. cinnamomi \\
\hline$P p I$ & 0.0002 & 0.0009 & 0.0081 & 0.0016 & 0.0629 & 0.0503 & 0.0534 & 0.0611 \\
\hline$P p I I$ & $\ldots$ & 0.0009 & 0.0079 & 0.0015 & 0.0627 & 0.0503 & 0.0533 & 0.0611 \\
\hline Pp III & $\ldots$ & $\ldots$ & 0.0088 & 0.0024 & 0.0638 & 0.0492 & 0.0522 & 0.0600 \\
\hline$P p I V$ & $\ldots$ & $\ldots$ & $\ldots$ & 0.0094 & 0.0712 & 0.0591 & 0.0627 & 0.0703 \\
\hline$P p V$ & $\ldots$ & $\ldots$ & $\ldots$ & $\ldots$ & 0.0636 & 0.0513 & 0.0550 & 0.0625 \\
\hline P. palmivora & $\ldots$ & $\ldots$ & $\ldots$ & $\ldots$ & $\ldots$ & 0.0434 & 0.0484 & 0.0583 \\
\hline P. tropicalis & $\ldots$ & $\ldots$ & $\ldots$ & $\ldots$ & $\ldots$ & $\ldots$ & 0.0429 & 0.0510 \\
\hline P. katsurae & $\ldots$ & $\ldots$ & $\ldots$ & $\ldots$ & $\ldots$ & $\ldots$ & $\ldots$ & 0.0511 \\
\hline
\end{tabular}

a PpI, PpII, PpIII, PpIV, and PpV are subgroups of Phytophthora nicotianae. The number of base substitutions per site from averaging over all sequence pairs within each group are shown. Analysis involved 128 nucleotide sequences. All positions containing gaps and missing data were eliminated. Evolutionary analyses were conducted in MEGA6. 
of more aggressive pathogens through intra- and interspecific cross hybridizations. Therefore, it is important to investigate population structure and genetic diversity of plant pathogens on ornamentals in Florida. Phytophthora spp. are one of the most frequently isolated pathogens from symptomatic ornamental crops in Florida (Merritt et al. 2012). Based on symptoms, the type of host species infected, and previous etiology, these isolates were tentatively assigned to previously described Phytophthora spp. Beyond these initial assessments, little was known about the genetic variation and population structure of this Phytophthora collection.

Cooke et al. (2000) conducted a phylogenetic study of Phytophthora spp. using ITS sequences. Moreover, a large number of ITS sequences of Phytophthora spp. are available to compare in GenBank. Phylogenetic analysis was based on the ITS sequences, a commonly used molecular marker, and a barcode for oomycetes, which provided sufficient resolution to interpret phylogenetic trees and to distinguish various Phytophthora spp. (Kroon et al. 2012; Martin et al. 2012; Robideau et al. 2011). Based on these analyses, this collection was separated into five groups (Fig. 1). Group 1 consisted of $P$. nicotianae, with the majority of isolates displaying very low SNP. Based on SNP data, the P. nicotianae group can be further divided into additional subgroups. These groups were not associated with a particular group of hosts, ruling out any strong host specificity among the isolates. Moreover, the tree branches defining these groups were not supported by strong bootstrap values $(<50 \%)$ and, therefore, each ITS sequence type has a broad host range. Our analyses, however, focused on species identification and it is likely that variation at other loci important for pathogenicity and aggressiveness, such as effector genes, could correlate with host range in $P$. nicotianae. With the ever-increasing ease and feasibility of sequencing entire genomes, this issue can be addressed by sequencing the entire genomes of select sets of isolates in this collection in the future. Analyses based on entire genome sequences will also

Table 4. Results of mating type testing ${ }^{\mathrm{a}}$

\begin{tabular}{lcccl}
\hline Species & \multicolumn{1}{c}{$\mathbf{A 1}$} & A2 & Sterile & A1A2 \\
\hline Phytophthora nicotianae & 28 & 40 & 22 & 0 \\
P. palmivora & 1 & 5 & 17 & 0 \\
P. tropicalis & 2 & 2 & 2 & 0 \\
P. katsurae & 0 & 0 & 0 & 3 \\
P. cinnamomi & 0 & 1 & 0 & 0 \\
Total & $31(25.2 \%)$ & $48(39.0 \%)$ & $41(33.3 \%)$ & $3(2.4 \%)$ \\
\hline
\end{tabular}

a Mating types were determined using know tester mating types A1 and A2; each isolate was also tested with itself for homothallism. Sterile isolates are defined as those that did not display any evidence of oospores, antheridia, or oogonia; A1A2 represents homothallic isolates. allow full-genome concatenated SNP-based analysis, which provides in-depth resolution in differentiating even closely related pathogen strains (Bart et al. 2012).

Evolutionary diversity estimates, which were as high as $7.5 \%$, indicate that this $P$. nicotianae population is highly diverse. The high divergence suggests that most of the isolates are not a single clonal lineage and that they were probably introduced along with their hosts into Florida. One of the most divergent isolates, 08-168, and several less divergent isolates were isolated from the same host (a Spathiphyllum sp.). This kind of host overlap can potentially provide a favorable breeding ground for hybridization and the emergence of new isolates containing novel complements of virulence factors, which can further expand their already wide host range. It is interesting to note that the first natural hybrid between two Phytophthora spp. was also isolated from a Spathiphyllum sp. (Man in 't Veld et al. 1998), suggesting that this plant and probably other tropical foliage plants are susceptible to multiple species of Phytophthora. Similarly, occurrence of several different Phytophthora spp. with so much variation within a small geographic location can also potentially lead to the emergence of novel hybrid species with adaptability to new environments and hosts (Leonberger et al. 2013). Several emerging pathogenic Phytophthora spp. such as P. andina and $P$. alni have been shown to be hybrids of different Phytophthora spp., which most likely coinhabited the same environment or even the same plant species (Brasier et al. 1999; Érsek and Nagy 2008; Goss et al. 2011a; Ioos et al. 2006). Similarly, hybrids between $P$. nicotianae (nicotianae) and $P$. cactorum, which possess an expanded host range, are likely due to a combination of novel virulent factors (Bonants et al. 2000; Hurtado-Gonzales et al. 2009; Man in 't Veld 2001; Man in 't Veld et al. 1998; Nirenberg et al. 2009). All these examples suggest that, if brought together, Phytophthora spp. can potentially hybridize, resulting in development of aggressive strains. This possibility is supported by the fact that both mating types A1 and A2 were represented in the isolates analyzed in this study. In our Phytophthora collection, we did not see any evidence of natural intra- or interspecific Phytophthora hybrids despite the fact that many isolates were recovered from a Spathiphyllum sp. A possible explanation could be incompatibility among Phytophthora spp. coinhabiting with the same individuals of a host plant species. For example, we recovered $P$. nicotianae and $P$. palmivora from Spathiphyllum spp. and, assuming that these two Phytophthora spp. are sympatric, their chances of hybridization might have been obscured by their genetic divergence. This is because $P$. nicotianae belongs to clade 1 and $P$. palmivora belongs to clade 4 according to recent molecular classification (Blair et al. 2008; Kroon et al. 2012). Similarly, P. tropicalis and P. palmivora both infect Hedera spp. and, thus, can potentially occur concurrently on the same individual plant and belong to distinct clades—clade 2 (P. tropicalis) and clade

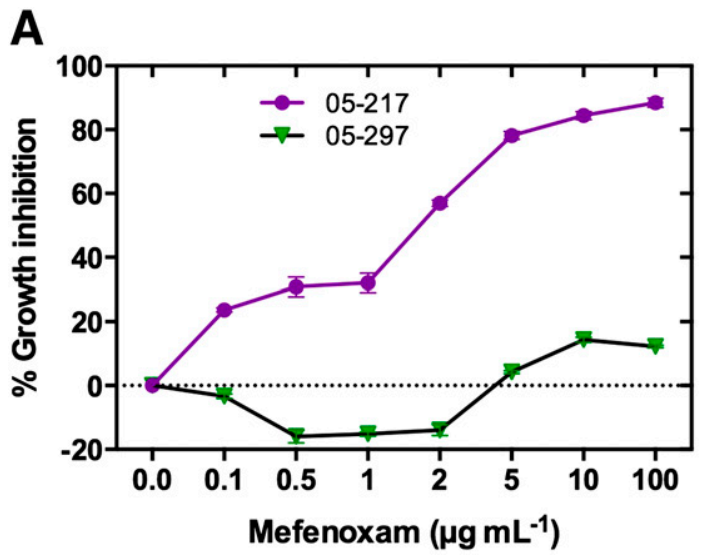

B

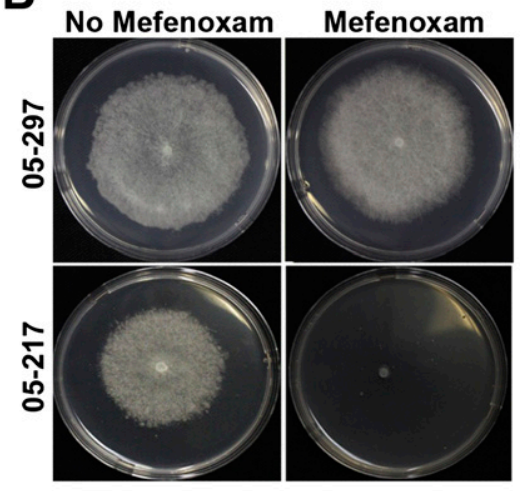

Fig. 2. Sensitivity of Phytophthora isolates to mefenoxam. A, Dose-response curves of Phytophthora nicotianae isolates 05-297 (resistant) and 05-217 (sensitive) to mefenoxam on V8 juice media. Each data point is a mean of four replications; error bars are standard error. B, Representative photographs of isolates $05-297$ and $05-217$ on mefenoxam -containing and control plates 5 days after the start of experiment. 
4 (P. palmivora) (Blair et al. 2008; Kroon et al. 2012)—suggesting that they also may be too genetically diverse to cross-hybridize. Evolutionary diversity estimates, which were as high as $7.5 \%$, indicate that this $P$. nicotianae population is highly divergent. The high divergence suggests that most of the isolates are not a single clonal lineage and that they were probably introduced along with their hosts into Florida. In our study, most isolates collected from 1993 to 2012 belong to mating type A2 whereas most isolates collected in 2013 belong to mating type A1. Further, most isolates collected in 2014 belong to mating type A2. Such a change in mating type year by year was evident in Europe for mating type A2 (Gisi and Cohen 1996). This pattern might involve epidemiological properties of the mating type, which is less competitive than the other mating type.

Assessing fungicides sensitivity of Phytophthora spp. is an essential step in planning and employing proper disease management strategies. Occurrence of fungicide resistance is a recurring problem with most single-site fungicides, including mefenoxam resistance in Phytophthora spp. (Ferrin and Kabashima 1991; Hu et al. 2005; Hu et al. 2008, 2010; Hwang and Benson 2005; Jeffers et al. 2004; Timmer et al. 1998), which can severely impact disease management programs. In several cases, mefenoxam resistance has been suggested to be associated with repeated use of mefenoxam (Hu et al. 2008). The isolates reported in this study were collected over a longer time from nurseries that rely on chemicals for disease control, thus raising the probability of fungicide resistance development. In our analyses, a majority of isolates were sensitive to all fungicides, including mefenoxam. This may be due to growers practicing fungicide rotation and tank mixes, which is widely publicized and emphasized through extension and outreach efforts in Florida. Moreover, only a few isolates (1 to 7) were collected each year starting from 1993, with exceptions of years 2013 and 2014. Such a small number of isolates collected each year could be a reason for the narrow range of species. For example, four species were recovered from a total of 45 isolates in 1993 to 2012 , whereas four species were recovered from a total of 78 isolates only from 2 years (2013 and 2014). However, there were notable exceptions of two isolates displaying insensitivity to mefenoxam. One isolate was recovered in 2005 and another isolate was recovered in 2014. In 2014, in total, 33 isolates were collected, which increased a chance of capturing resistant isolates. This was expected because mefenoxam has been used extensively in nurseries in Florida. The substantial variation in mefenoxam sensitivities in tested isolates suggests that they are still evolving. It is likely that these isolates could further evolve and become more resistant to mefenoxam, especially if mefenoxam is used exclusively. These results agree with those reported by Ferrin and Kabashima (1991), where 7.7\% of the $P$. nicotianae isolates collected from nurseries in Southern California were mefenoxam resistant (Ferrin and Kabashima 1991). Our results differed substantially from those reported from $P$. nicotianae isolates collected from nurseries elsewhere, which reported $21 \%$ (North Carolina) and 26\% (Virginia) mefenoxam-resistant isolates (Hu et al. 2008; Hwang and Benson 2005). Hu et al. (2008) collected 95 isolates of $P$. nicotianae from diverse nursery crops and irrigation water from Virginia, whereas Hwang and Benson (2005) recovered 483 isolates from seven different plant species from North Carolina. Based on our data, if we assume that approximately $8.8 \%$ P. nicotianae isolates present in Florida are resistant to mefenoxam and that mefenoxam is used frequently, we should have seen an increase of mefenoxam-resistant isolates over time. However, this is not what was observed in the analyses. The most resistant isolate (05-297) was isolated in 2005, whereas isolates collected after 2005 were more sensitive to mefenoxam. An explanation for this is that the plants could have been shipped elsewhere before Phytophthora spp. could become established in the nursery, or they could have been phased out from natural populations due to use of fungicides with modes of actions that are different from mefenoxam. In addition, mefenoxam has been in the market since 1996 for use on various crops but, as years have passed, more fungicides have become available which belong to different modes of action. Commercialization of new products may have reduced the exposure of Phytophthora spp. to mefenoxam. More intensive and extensive sampling might be needed to gain a true assessment of the prevalence of mefenoxam-resistant isolates in Florida.

Our analysis finds resistance in Phytophthora spp. to Adorn (fluopicolide), Aliette (fosetyl-Al), Heritage (azoxystrobin), and Segway (cyazofamid). To our knowledge, this is the first report of resistance in an isolate of Phytophthora to fluopicolide. The finding of an isolate resistant to Adorn is not surprising because fluopicolide has a single-site mode of action for efficacy against oomycetes. In fact, the product manufacturer recommends that Adorn is not to be applied alone. There are reports of reduced sensitivity in Phytophthora spp. to Aliette. For example, P. cinnamomi obtained from avocado trees continuously treated with phosphonates was less inhibited by Aliette (Duvenhage 1994). Similarly, all $P$. capsici isolates collected from diverse vegetable crops were found to have either resistance or intermediate sensitivity to Segway (Jackson et al. 2012).

Several groups have reported increased fitness of metalaxylresistant isolates as increased vegetative growth, oospore formation, and lesion sizes (Hu et al. 2008; Kadish and Cohen 1988a,b; Kadish et al. 1990; Porter et al. 2007). Interestingly, in our study, we found that, in the presence of lower mefenoxam doses, mefenoxamresistant isolates displayed increased mycelial growth, suggesting that these isolates have adapted to exploit mefenoxam to their advantage through an unknown mechanism. Because mefenoxam is suggested to affect the activity of RNA polymerases (Davidse et al. 1983, 1988; Fisher and Hayes 1984), it is possible that the resistant isolates have accumulated mutations that, instead of inhibiting, enhance the activity of RNA polymerase for favorable mycelial growth. Mefenoxam has been popular for controlling Phytophthora diseases in ornamental nurseries. The frequencies of resistant $P$. nicotianae isolates may increase in the future if these isolates have greater fitness compared with sensitive isolates. Moreover, by cross hybridizing with other Phytophthora isolates or species, mefenoxam resistance can be transferred into hybrids with better fitness in natural ecosystems. Therefore, caution is advised in using mefenoxam where it has been found to be ineffective; under such situations, it is advisable to discontinue using mefenoxam altogether because this can lead to the selection and reappearance of mefenoxam-resistant populations, as was suggested for $P$. infestans (Hu et al. 2012). Resistance can also be managed by rotating fungicides with different modes of action such as fosetyl-Al and dimethomorph, which provided effective control against those isolates, which were resistant to mefenoxam. In addition, all other possible integrated disease management approaches should be practiced such as using clean potting mixes, clean water, and including biological and biorational pesticides with broad-spectrum activity. This work provides important information about the genetic structure of Phytophthora populations in Florida. These findings will help in formulating effective strategies and policies for preventing the spread of Phytophthora spp. to new niches and will mitigate risks of fungicide resistance development.

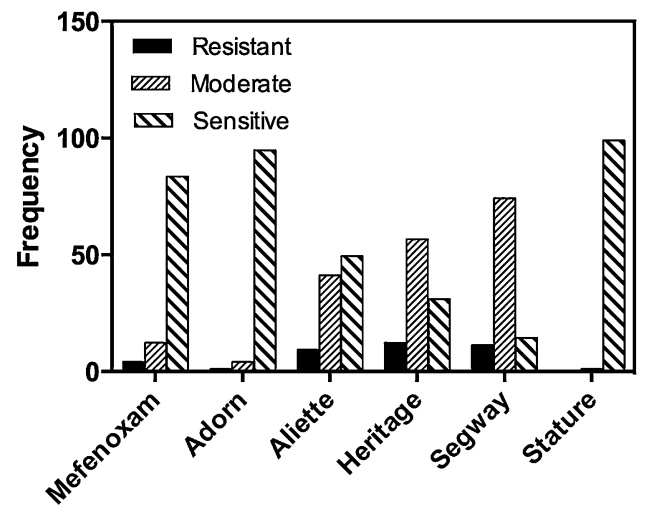

Fig. 3. Frequency distribution of resistant, moderately resistant, and sensitive Phytophthora isolates to different fungicides. Resistant, $\leq 50 \%$ growth inhibition; Moderate, moderately resistant, with 51 to $79 \%$ growth inhibition; and Sensitive, $\geq 80 \%$ growth inhibition. 


\section{Acknowledgments}

This work was supported by funds from the Institute of Food and Agricultural Sciences at the University of Florida to G. S. Ali. We thank R. Ali for her help with mating typing analyses and fungicide resistance assays.

\section{Literature Cited}

Aljanabi, S. M., and Martinez, I. 1997. Universal and rapid salt-extraction of high quality genomic DNA for PCR-based techniques. Nucleic Acids Res. 25: 4692-4693.

Bart, R., Cohn, M., Kassen, A., McCallum, E. J., Shybut, M., Petriello, A., Krasileva, K., Dahlbeck, D., Medina, C., Alicai, T., Kumar, L., Moreira, L. M., Rodrigues Neto, J., Verdier, V., Santana, M. A., Kositcharoenkul, N., Vanderschuren, H., Gruissem, W., Bernal, A., and Staskawicz, B. J. 2012. High-throughput genomic sequencing of cassava bacterial blight strains identifies conserved effectors to target for durable resistance. Proc. Natl. Acad. Sci. USA 109:E1972-E1979.

Bienapfl, J. C., and Balci, Y. 2014. Movement of Phytophthora spp. in Maryland's nursery trade. Plant Dis. 98:134-144.

Blair, J. E., Coffey, M. D., Park, S. Y., Geiser, D. M., and Kang, S. 2008. A multilocus phylogeny for Phytophthora utilizing markers derived from complete genome sequences. Fungal Genet. Biol. 45:266-277.

Bonants, P. J., Hagenaar-de Weerdt, M., Man in 't Veld, W. A., and Baayen, R. P. 2000. Molecular characterization of natural hybrids of Phytophthora nicotianae and P. cactorum. Phytopathology 90:867-874.

Brasier, C. M., Cooke, D. E., and Duncan, J. M. 1999. Origin of a new Phytophthora pathogen through interspecific hybridization. Proc. Natl. Acad. Sci. USA 96:5878-5883.

Brent, K. 1992. Monitoring fungicide resistance: Purposes, procedures and progress. Pages 1-18 in: Resistance '91: Achievements and Developments in Combating Pesticide Resistance. I. Denholm, A. Devonshire, and D. Hollomon, eds. Elsevier, London and New York.

Cacciola, S. O., and Lio, G. M. S. 2008. Management of citrus diseases caused by Phytophthora spp. Pages 61-84 in: Integrated Management of Diseases Caused by Fungi, Phytoplasma and Bacteria. A. Ciancio and K. G. Mukerji, eds. Springer, The Netherlands.

Cline, E. T., Farr, D. F., and Rossman, A. Y. 2008. A synopsis of Phytophthora with accurate scientific names, host range, and geographic distribution. Online publication. Plant Health Prog. doi:10.1094/PHP-20080318-01-RS.

Cooke, D. E., Drenth, A., Duncan, J. M., Wagels, G., and Brasier, C. M. 2000. A molecular phylogeny of Phytophthora and related oomycetes. Fungal Genet. Biol. 30:17-32.

Davidse, L. C., Gerritsma, O. C. M., Ideler, J., Pie, K., and Velthuis, G. C. M. 1988. Antifungal modes of action of metalaxyl, cyprofuram, benalaxyl and oxadixyl in phenylamide-sensitive and phenylamide-resistant strains of Phytophthora megasperma f. sp. medicaginis and Phytophthora infestans. Crop Prot. 7: 347-355.

Davidse, L. C., Hofman, A. E., and Velthuis, G. C. M. 1983. Specific interference of metalaxyl with endogenous RNA polymerase activity in isolated nuclei from Phytophthora megasperma f. sp. medicaginis. Exp. Mycol. 7: 344-361.

Dobrowolski, M. P., Shearer, B. L., Colquhoun, I. J., O'Brien, P. A., and Hardy, G. E. S. 2008. Selection for decreased sensitivity to phosphite in Phytophthora cinnamomi with prolonged use of fungicide. Plant Pathol. 57:928-936.

Duvenhage, J. A. 1994. Monitoring the resistance of Phytophthora cinnamomi to fosetyl-AI and H3PO3. South African Avocado Growers' Association Yearbook 17:35-37.

DPI. 1998. Certified Nursery Directory. Florida Department of Agriculture and Consumer Services, Gainesville, FL.

Edgar, R. C. 2004. MUSCLE: Multiple sequence alignment with high accuracy and high throughput. Nucleic Acids Res. 32:1792-1797.

Érsek, T., and Nagy, Z. A. 2008. Species hybrids in the genus Phytophthora with emphasis on the alder pathogen Phytophthora alni: A review. Eur. J. Plant Pathol. 122:31-39.

Erwin, D. C., and Ribeiro, O. K. 1996. Phytophthora Diseases Worldwide. American Phytopathological Society, St. Paul, MN.

Ferrin, D. M., and Kabashima, J. N. 1991. In vitro insensitivity to metalaxyl of isolates of Phytophthora citricola and P. parasitica from ornamental hosts in southern California. Plant Dis. 75:1041-1044.

Fisher, D. J., and Hayes, A. L. 1984. Studies of mechanisms of metalaxyl fungitoxicity and resistance to metalaxyl. Crop Prot. 3:177-185.

Foster, J. M., and Hausbeck, M. K. 2010. Managing Phytophthora crown and root rot in bell pepper. Plant Dis. 94:697-702.

FRAC. 2015. FRAC CodeList: Fungicides sorted by mode of action. Fungicide Resistance Action Committee. Online publication. http://www.frac. info/publications/

Gevens, A. J., Donahoo, R. S., Lamour, K. H., and Hausbeck, M. K. 2007. Characterization of Phytophthora capsici from Michigan surface irrigation water. Phytopathology 97:421-428.

Gisi, U., and Cohen, Y. 1996. Resistance to phenylamide fungicides: A case study with Phytophthora infestans involving mating type and race structure. Annu. Rev. Phytopathol. 34:549-572.
Goss, E. M., Cardenas, M. E., Myers, K., Forbes, G. A., Fry, W. E., Restrepo, S and Grünwald, N. J. 2011a. The plant pathogen Phytophthora andina emerged via hybridization of an unknown Phytophthora species and the Irish potato famine pathogen, $P$. infestans. PLoS One 6:e24543.

Goss, E. M., Larsen, M., Vercauteren, A., Werres, S., Heungens, K., and Grünwald, N. J. 2011b. Phytophthora ramorum in Canada: Evidence for migration within North America and from Europe. Phytopathology 101: 166-171.

Grünwald, N. J., Garbelotto, M., Goss, E. M., Heungens, K., and Prospero, S 2012. Emergence of the sudden oak death pathogen Phytophthora ramorum. Trends Microbiol. 20:131-138.

Grünwald, N. J., Goss, E. M., and Press, C. M. 2008. Phytophthora ramorum A pathogen with a remarkably wide host range causing sudden oak death on oaks and ramorum blight on woody ornamentals. Mol. Plant Pathol. 9:729-740.

Grünwald, N. J., Martin, F. N., Larsen, M. M., Sullivan, C. M., Press, C. M., Coffey, M. D., Hansen, E. M., and Parke, J. L. 2011. Phytophthora-ID.org: A sequence-based Phytophthora identification tool. Plant Dis. 95:337-342.

Grünwald, N. J., Sturbaum, A. K., Montes, G. R., Serrano, E. G., Lozoya-Saldana, H., and Fry, W. E. 2006. Selection for fungicide resistance within a growing season in field populations of Phytophthora infestans at the center of origin. Phytopathology 96:1397-1403.

Hu, C.-H., Perez, F. G., Donahoo, R., McLeod, A., Myers, K., Ivors, K., Secor, G. Roberts, P. D., Deahl, K. L., Fry, W. E., and Ristaino, J. B. 2012. Recent genotypes of Phytophthora infestans in the eastern United States reveal clonal populations and reappearance of mefenoxam sensitivity. Plant Dis. 96: 1323-1330.

Hu, J., Stromberg, E., Moorman, G., and Hong, C. 2005. Mefenoxam and propamocarb sensitivity of Phytophthora cinnamomi at ornamental plan nurseries in Virginia. (Abstr.) Phytopathology 95:S45.

Hu, J. H., Hong, C. X., Stromberg, E. L., and Moorman, G. W. 2008. Mefenoxam sensitivity and fitness analysis of Phytophthora nicotianae isolates from nurseries in Virginia, USA. Plant Pathol. 57:728-736.

Hu, J. H., Hong, C. X., Stromberg, E. L., and Moorman, G. W. 2010. Mefenoxam sensitivity in Phytophthora cinnamomi isolates. Plant Dis. 94:39-44.

Hurtado-Gonzales, O. P., Aragon-Caballero, L. M., Flores-Torres, J. G., in 't Veld, W. M., and Lamour, K. H. 2009. Molecular comparison of natural hybrids of Phytophthora nicotianae and P. cactorum infecting loquat trees in Peru and Taiwan. Mycologia 101:496-502.

Hwang, J., and Benson, D. M. 2005. Identification, mefenoxam sensitivity, and compatibility type of Phytophthora spp. attacking floriculture crops in North Carolina. Plant Dis. 89:185-190.

Ioos, R., Andrieux, A., Marcais, B., and Frey, P. 2006. Genetic characterization of the natural hybrid species Phytophthora alni as inferred from nuclear and mitochondrial DNA analyses. Fungal Genet. Biol. 43:511-529.

Jackson, K. L., Yin, J. F., Csinos, A. S., and Ji, P. S. 2010. Fungicidal activity of fluopicolide for suppression of Phytophthora capsici on squash. Crop Prot. 29: 1421-1427.

Jackson, K. L., Yin, J., and Ji, P. 2012. Sensitivity of Phytophthora capsici on vegetable crops in Georgia to Mandipropamid, dimethomorph, and cyazofamid. Plant Dis. 96:1337-1342.

Jeffers, S. N., Schnabel, G., and Smith, J. P. 2004. First report of resistance to mefenoxam in Phytophthora cactorum in the United States and elsewhere. Plant Dis. 88:576.

Kadish, D., and Cohen, Y. 1988a. Non-competitive fitness of metalaxyl-sensitive and metalaxyl-resistant populations of Phytophthora infestans. Phytoparasitica $16: 80-81$

Kadish, D., and Cohen, Y. 1988b. Fitness of Phytophthora infestans isolates from metalaxyl-sensitive and metalaxyl-resistant populations. Phytopathology 78: 912-915.

Kadish, D., Grinberger, M., and Cohen, Y. 1990. Fitness of metalaxyl-sensitive and metalaxyl-resistant isolates of Phytophthora infestans on susceptible and resistant potato cultivars. Phytopathology 80:200-205.

Kamoun, S., Furzer, O., Jones, J. D., Judelson, H. S., Ali, G. S., Dalio, R. J., Roy, S. G., Schena, L., Zambounis, A., Panabieres, F., Cahill, D., Ruocco, M, Figueiredo, A., Chen, X. R., Hulvey, J., Stam, R., Lamour, K., Gijzen, M. Tyler, B. M., Grünwald, N. J., Mukhtar, M. S., Tome, D. F., Tor, M., Van den Ackerveken, G., McDowell, J., Daayf, F., Fry, W. E., Lindqvist-Kreuze, H., Meijer, H. J., Petre, B., Ristaino, J., Yoshida, K., Birch, P. R., and Govers, F. 2015. The top 10 oomycete pathogens in molecular plant pathology. Mol. Plant Pathol. 16:413-443.

Kang, S., Park, J., Park, B., Blair, J., Geiser, D., Makalowska, I., Park, S., Coffey, M., Green, M., Ivors, K., Lee, Y., O'Donnell, K., and Martin, F. 2007. Phytophthora database: A cyber-infrastructure for detecting, monitoring, and managing Phytophthora. (Abstr.) Phytopathology 97:S157.

Kousik, C. S., and Keinath, A. P. 2008. First report of insensitivity to cyazofamid among isolates of Phytophthora capsici from the southeastern United States. Plant Dis. 92:979.

Kroon, L. P., Brouwer, H., de Cock, A. W., and Govers, F. 2012. The genus Phytophthora anno 2012. Phytopathology 102:348-364.

Kuhajek, J. M., Jeffers, S. N., Slattery, M., and Wedge, D. E. 2003. A rapid microbioassay for discovery of novel fungicides for Phytophthora spp. Phytopathology 93:46-53. 
Lamour, K. H., and Hausbeck, M. K. 2000. Mefenoxam insensitivity and the sexual stage of Phytophthora capsici in Michigan cucurbit fields. Phytopathology 90:396-400.

Larkin, M. A., Blackshields, G., Brown, N. P., Chenna, R., McGettigan, P. A., McWilliam, H., Valentin, F., Wallace, I. M., Wilm, A., Lopez, R., Thompson, J. D., Gibson, T. J., and Higgins, D. G. 2007. Clustal W and Clustal X version 2.0. Bioinformatics 23:2947-2948.

Lee, S. B., and Taylor, J. W. 1992. Phylogeny of five fungus-like protoctistan Phytophthora species, inferred from the internal transcribed spacers of ribosomal DNA. Mol. Biol. Evol. 9:636-653.

Leonberger, A. J., Speers, C., Ruhl, G., Creswell, T., and Beckerman, J. L. 2013. A survey of Phytophthora spp. in Midwest nurseries, greenhouses, and landscapes. Plant Dis. 97:635-640.

Man in 't Veld, W. A. 2001. First report of natural hybrids of Phytophthora nicotianae and $P$. cactorum on loquat in Taiwan. Plant Dis. 85:98.

Man in 't Veld, W. A., Veenbaas-Rijks, W. J., Ilieva, E., de Cock, A. W., Bonants, P. J., and Pieters, R. 1998. Natural hybrids of Phytophthora nicotianae and Phytophthora cactorum demonstrated by isozyme analysis and random amplified polymorphic DNA. Phytopathology 88:922-929.

Martin, F. N., Abed, Z. G., Baldi, Y., and Ivors, K. 2012. Identification and detection of Phytophthora: Reviewing our progress, identifying our needs. Plant Dis. 96:1080-1103.

Meng, Q. X., Cui, X. L., Bi, Y., Wang, Q., Hao, J. J., and Liu, X. L. 2011. Biological and genetic characterization of Phytophthora capsici mutants resistant to flumorph. Plant Pathol. 60:957-966.

Merritt, J. L., Dickstein, E., Johnson, R. S., Ward, M., Balaam, R. J., Harmon, C. L., Harmon, P. F., Ali, G. S., Palmateer, A. J., Schubert, T., and van Bruggen, A. H. C. 2012. Survey of ornamental nurseries in Florida participating in the U S-Canadian Greenhouse Certification Program. HortTechnology 22:169-176.

Nirenberg, H. I., Gerlach, W. F., and Grafenhan, T. 2009. Phytophthora $\times$ pelgrandis, a new natural hybrid pathogenic to Pelargonium grandiflorum hort. Mycologia 101:220-231.

Park, J., Park, B., Veeraraghavan, N., Jung, K., Lee, Y. H., Blair, J. E., Geiser, D. M., Isard, S., Mansfield, M. A., Nikolaeva, E., Park, S. Y., Russo, J., Kim, S. H., Greene, M., Ivors, K. L., Balci, Y., Peiman, M., Erwin, D. C., Coffey, M. D., Rossman, A., Farr, D., Cline, E., Grünwald, N. J., Luster, D. G., Schrandt, J., Martin, F., Ribeiro, O. K., Makalowska, I., and Kang, S. 2008. Phytophthora database: A forensic database supporting the identification and monitoring of Phytophthora. Plant Dis. 92:966-972.

Parke, J. L., Knaus, B. J., Fieland, V. J., Lewis, C., and Grünwald, N. J. 2014. Phytophthora community structure analyses in Oregon nurseries inform systems approaches to disease management. Phytopathology 104:1052-1062.

Pérez-Sierra, A., Alvarez, L. A., Vercauteren, A., Heungens, K., and AbadCampos, P. 2011. Genetic diversity, sensitivity to phenylamide fungicides and aggressiveness of Phytophthora ramorum on Camellia, Rhododendron and Viburnum plants in Spain. Plant Pathol. 60:1069-1076.

Porter, L. D., Miller, J. S., Nolte, P., and Price, W. J. 2007. In vitro somatic growth and reproduction of phenylamide-resistant and -sensitive isolates of Phytophthora erythroseptica from infected potato tubers in Idaho. Plant Pathol. 56:492-499.
Prigigallo, M. I., Mosca, S., Cacciola, S. O., Cooke, D. E. L., and Schena, L. 2015. Molecular analysis of Phytophthora diversity in nursery-grown ornamental and fruit plants. Plant Pathol. 64:1308-1319.

Prospero, S., Grünwald, N. J., Winton, L. M., and Hansen, E. M. 2009. Migration patterns of the emerging plant pathogen Phytophthora ramorum on the west coast of the United States of America. Phytopathology 99:739-749.

Rizzo, D. M., Garbelotto, M., Davidson, J. M., Slaughter, G. W., and Koike, S. T. 2002. Phytophthora ramorum as the cause of extensive mortality of Quercus spp. and Lithocarpus densiflorus in California. Plant Dis. 86:205-214.

Robideau, G. P., De Cock, A. W., Coffey, M. D., Voglmayr, H., Brouwer, H., Bala, K., Chitty, D. W., Desaulniers, N., Eggertson, Q. A., Gachon, C. M., Hu, C. H., Kupper, F. C., Rintoul, T. L., Sarhan, E., Verstappen, E. C., Zhang, Y., Bonants, P. J., Ristaino, J. B., and Levesque, C. A. 2011. DNA barcoding of oomycetes with cytochrome c oxidase subunit I and internal transcribed spacer. Mol. Ecol. Resour. 11:1002-1011

Schlenzig, A., Campbell, R., and Chard, J. 2015. Phytophthora species infecting hardy ornamentals in nurseries and the managed environment in Scotland. J. Phytopathol. 163:686-689.

Staub, T., and Sozzi, D. 1984. Fungicide resistance - a continuing challenge. Plant Dis. 68:1026-1031

Stein, J. M., and Kirk, W. W. 2004. The generation and quantification of resistance to dimethomorph in Phytophthora infestans. Plant Dis. 88:930-934.

Tamura, K., and Nei, M. 1993. Estimation of the number of nucleotide substitutions in the control region of mitochondrial DNA in humans and chimpanzees. Mol. Biol. Evol. 10:512-526.

Tamura, K., Nei, M., and Kumar, S. 2004. Prospects for inferring very large phylogenies by using the neighbor-joining method. Proc. Natl. Acad. Sci. USA 101:11030-11035.

Tamura, K., Peterson, D., Peterson, N., Stecher, G., Nei, M., and Kumar, S. 2011. MEGA5: Molecular evolutionary genetics analysis using maximum likelihood, evolutionary distance, and maximum parsimony methods. Mol. Biol. Evol. 28: 2731-2739.

Timmer, L. W., Graham, J. H., and Zitko, S. E. 1998. Metalaxyl-resistant isolates of Phytophthora nicotianae: Occurrence, sensitivity, and competitive parasitic ability on citrus. Plant Dis. 82:254-261.

Wang, Y., Bouwmeester, K., van de Mortel, J. E., Shan, W., and Govers, F. 2013. A novel Arabidopsis-oomycete pathosystem; differential interactions with Phytophthora capsici reveal a role for camalexin, indole glucosinolates and salicylic acid in defense. Plant Cell Environ. 36:1192-1203.

Wawra, S., Belmonte, R., Lobach, L., Saraiva, M., Willems, A., and van West, P. 2012. Secretion, delivery and function of oomycete effector proteins. Curr. Opin. Microbiol. 15:685-691.

White, T. J., Bruns, T., Lee, S., and Taylor, J. 1990. Amplification and direct sequencing of fungal ribosomal RNA genes for phylogenetics. Pages 315-322 in: PCR Protocols: A Guide to Methods and Applications. Academic Press, San Diego, CA.

Ziogas, B. N., Markoglou, A. N., Theodosiou, D. I., Anagnostou, A., and Boutopoulou, S. 2006. A high multi-drug resistance to chemically unrelated oomycete fungicides in Phytophthora infestans. Eur. J. Plant Pathol. 115:283-292. 